\title{
The vibration control research of MRE damper for the multi-level eccentric structure
}

\author{
Jiayun Xu Changsheng Wang Lihang Wang Leiyu Cao \\ Hubei Key Laboratory of Road Bridge and Structural Engineering, Institute of Civil Engineering and \\ Architecture, Wuhan University of Technology, Wuhan 430070, China \\ wangchangshenghao@163.com
}

Keywords: Eccentric structure; The translation-torsion coupling; MRE damper; Torsional vibration; Vibration control

\begin{abstract}
For the characteristics of that the center of mass and stiffness centre do not overlap and that presence of translational and torsional vibrations under earthquake to the eccentric structure, MRE damper is used to control the translation-torsion coupling effect of eccentric structure under seismic action. Comparative analysis the structural vibration response in the absence of control and without control. Numerical calculation shows that the translational and torsional vibration damping ratio of the structure under the effect of control can reach maximum of $70.41 \%$ and $26.13 \%$ respectively. Thus illustrates the MRE damper for vibration control of eccentric structure is effective.
\end{abstract}

\section{Introduction}

Due to the irregular structure layout, the center of mass and stiffness centre do not overlap, resulting the translational vibration and torsional vibration under earthquake (Dang et al.2010). All previous earthquake damage and theoretical analysis show that the ratio of structure torsional vibration damage is obvious of all forms of damage (Xiao et al.2012). So the torsional vibration response and effect control is being focus on. Traditional design method that is to increase the stiffness of the structure and adjust the structure to reduce the torsional vibration of the structure can not solve the problem of the vibration response of eccentric structure (Tang et al.2008). Nowadays the mature method is to set up control devices in structure to reduce the vibration of the structure. MRE is the solid simulation of MRF, mainly composed by matrix materials and dispersing magnetic particles. That use magneto-rheological solid material can solve the problem of magnetic particle settlement, MRE device has advantages of no need to seal, stable performance, easy to control with quick response etc ( $\mathrm{Ni}$ et al.2010; $\mathrm{Tu}$ et al.2003; Long et al.2013). So the magneto-rheological dampers are widely used in engineering. In this paper, installing MRE dampers on the eccentric structure to absorb vibration energy, thereby reducing translational and torsional vibration under earthquake.

\section{Structure Equations of Motion}

For multilayer or high level biaxial eccentric structure under the seismic action, the translation-torsion coupling equations of motion of the structure is as follows:

$$
M \Phi(t)+C X(t)+K X(t)=-M I \frac{1}{g}(t)
$$

where $\boldsymbol{M} 、 \boldsymbol{K} 、 \boldsymbol{C}$ were the mass matrix 、 stiffness matrix 、 damping matrix of structure. $\boldsymbol{x}(t)$ is the displacement column vector of structure; $I=\{1,1 \mathrm{~L} 1 ; \lambda, \lambda \mathrm{L} \lambda ; 0,0 \mathrm{~L} 0\}^{T}, \lambda$ is the ratio of seismic wave peak acceleration to $\mathrm{x}$ and $\mathrm{y}$ direction. $(t)$ is the input seismic acceleration; Using Rayleigh damping assumption to set up the structural damping matrix of structural system, $\boldsymbol{C}=\alpha_{0} \boldsymbol{M}+\alpha_{1} \boldsymbol{K}$ in the equation (1), where the expressions of the coefficient $\alpha_{0} 、 \alpha_{1}$ are obtained by the formula(Liu et al.2005): $\xi_{n}=\alpha_{0} / 2 \omega_{n}+\alpha_{1} \omega_{n} / 2$. Given any two modal damping ratio $\xi$ (Since the oscillation frequency can be obtained by mass matrix and stiffness matrix), we can get the scale factor $\alpha_{0}$ and $\alpha_{1}$. 


$$
\begin{aligned}
& \boldsymbol{M}=\left[\begin{array}{lll}
\boldsymbol{m} & & \\
& \boldsymbol{m} & \\
& & \boldsymbol{J}
\end{array}\right], \boldsymbol{m}=\left[\begin{array}{llll}
m_{1} & & & \\
& m_{2} & & \\
& & \circ & \\
& & & m_{n}
\end{array}\right], \boldsymbol{J}=\left[\begin{array}{llll}
J_{1} & & & \\
& J_{2} & & \\
& & \circ & \\
& & & J_{n}
\end{array}\right], \boldsymbol{K}=\left[\begin{array}{llll}
\boldsymbol{k}_{x x} & & & \boldsymbol{k}_{x \varphi} \\
& \boldsymbol{k}_{y y} & \boldsymbol{k}_{y \varphi} \\
\boldsymbol{k}_{\varphi x} & \boldsymbol{k}_{\varphi y} & \boldsymbol{k}_{\varphi \varphi}
\end{array}\right],
\end{aligned}
$$

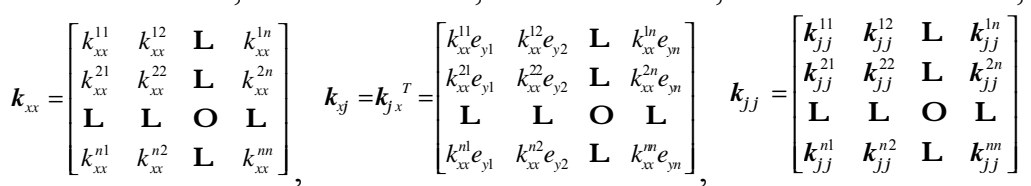

Where $\boldsymbol{k}_{y y}$ and $\boldsymbol{k}_{y \varphi}=\boldsymbol{k}_{\varphi y}{ }^{T}$ are similar to $\boldsymbol{k}_{x x}$ and $\boldsymbol{k}_{x \varphi}=\boldsymbol{k}_{\varphi x}{ }^{T}$ in the expression, Just to exchange the subscript of the $\mathrm{x}$ and $\mathrm{y} . e_{x i} 、 e_{y i}$ are respectively the $\mathrm{i}$-th layer $\mathrm{x}$ and $\mathrm{y}$ direction of eccentricity.

\section{Using MRE Damper to Structure Control}

For the characteristics that shear modulus control is strong and the damping coefficient changes little of the magneto-rheological elastomers, the selected magneto-rheological elastomers mechanical model is a viscoelastic model which attached a magnetic shear modulus on the basis of Calvin model, as shown in figure 1.the magneto-rheological elastomer shear modulus in the model consists of two parts: the inherent shear modulus without magnetic field and magnetic shear modulus caused by magnetic field(Fang et al.2004; Fu et al.2013).

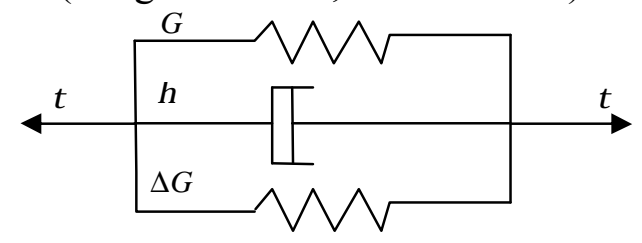

Fig. 1 MRE mechanical model

The output force calculating formula of the magneto-rheological elastomer damper:

$$
F=k_{0} x+\Delta k x+c \&=\left(k_{0}+\Delta k\right) x+c \&
$$

Where $k_{0}=G A / h, G$ is the inherent shear modulus without magnetic field, $A$ is The area of the magneto-rheological elastomers, $h$ is the thickness of the magneto-rheological elastomers. $\Delta k=\Delta G A / h, \Delta G$ is the magnetic shear modulus caused by magnetic field. $c=\eta A / h, c$ is the damping coefficient of magneto-rheological elastomers dampers, $\eta=2 \zeta$.Control system state equation:

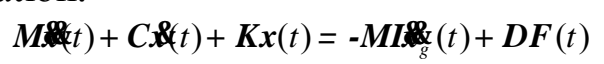

Where $\boldsymbol{D}$ is the position matrix of control force, $\boldsymbol{F}(t)$ is the control force vector.

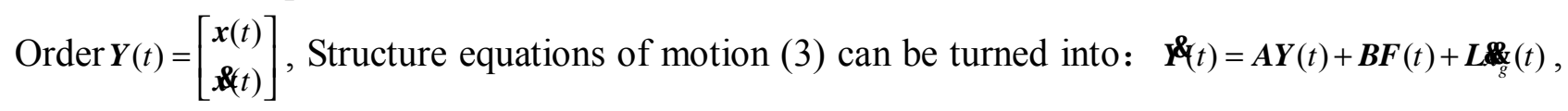
where $\boldsymbol{A}=\left[\begin{array}{cc}\boldsymbol{0}_{n \times n} & \boldsymbol{I}_{n} \\ -\boldsymbol{M}^{-1} \boldsymbol{K} & -\boldsymbol{M}^{-1} \boldsymbol{C}\end{array}\right] ; \boldsymbol{B}=\left[\begin{array}{c}\boldsymbol{0}_{n \times n} \\ \boldsymbol{M}^{-1} \boldsymbol{D}\end{array}\right] ; \boldsymbol{L}=\left[\begin{array}{c}\boldsymbol{0}_{n \times 1} \\ -\boldsymbol{I}\end{array}\right]$

\section{Control Strategy}

Optimal control is that under certain conditions, to determine an optimal control strategy for a motion system, which the initial state of the motion system shifts to a predetermined target state, and performance indicators to achieve the optimal value. LQR (Liner quadratic regulator) control is an optimal control of linear quadratic optimal control, which is the optimal control of the most widely used, most highly developed control. The control object of LQR control is the linear motion systems in the form of spatial state, whose function is a quadratic function of the amount of the performance index $\mathrm{Y}(\mathrm{t})$ and control the amount of $\mathrm{F}(\mathrm{t})$. In the linear quadratic optimal control, the selected control force vector $\mathrm{F}(\mathrm{t})$ is to make performance index $\mathrm{J}$ to the minimum $(\mathrm{Xu}$ et al.2007; Zhou.2014). The performance index $\mathrm{J}$ is usually taken by: 


$$
J=\frac{1}{2} \int_{0}^{t_{f}}\left(\boldsymbol{Y}^{T}(t) \boldsymbol{Q Y}(t)+\boldsymbol{F}^{T}(t) \boldsymbol{R} \boldsymbol{F}(t)\right) d t
$$

Where $\boldsymbol{Q}$ is Semi-definite weighting matrix, $\boldsymbol{R}$ is definite weighting matrix. Applicated of Minimax control principle, the optimal control force to make the performance index $\mathrm{J}$ to the minimum is as follows:

$$
\boldsymbol{F}(t)=-\boldsymbol{R}^{-1} \boldsymbol{B}^{T} \boldsymbol{P} \boldsymbol{Y}(t)
$$

Where $\boldsymbol{P}$ is the Riccati matrix, which can be obtained by the Riccati equation $\boldsymbol{P A}+\boldsymbol{A}^{T} \boldsymbol{P}-\boldsymbol{P} \boldsymbol{B} \boldsymbol{R}^{-1} \boldsymbol{B}^{T} \boldsymbol{P}+\boldsymbol{Q}=0 . \boldsymbol{Q} 、 \boldsymbol{R}$ are usually taken as a diagonal matrix:

$$
\boldsymbol{Q}=\alpha\left[\begin{array}{cc}
\boldsymbol{K} & 0 \\
0 & \boldsymbol{M}
\end{array}\right] \quad \boldsymbol{R}=\beta \boldsymbol{I}
$$

Where $\alpha, \beta$ can be obtained by the trial of the control system. (Select $\alpha=100, \beta=6.5 \times 10^{-5}$ ).

\section{Numerical Analysis}

In order to discuss the control effect of the structure at MRE damper effect, we select an eight-story frame structure to analysis. The mass of each layer: $\boldsymbol{m}=[865,865,840,840,815,805,785,760] \times 10^{3} \mathrm{~kg}$, the horizontal stiffness to $\mathrm{X}$ direction of each layer: $\boldsymbol{k}_{x}=[145,145,132,132,121,110,105,95] \times 10^{6} \mathrm{~N} / \mathrm{m}$, the horizontal stiffness to $\mathrm{Y}$ direction of each layer: $\boldsymbol{k}_{y}=[125,125,112,112,105,95,86,75] \times 10^{6} \mathrm{~N} / \mathrm{m}$, the eccentricity of each layer: $e_{x}=e_{y}=2.0 m$. We installed a magneto-rheological elastomer damper on each layer in the structure, and take EI Centro seismic waves of a peak at $200 \mathrm{~cm} / \mathrm{s}^{2}$ as the input seismic waves. Through the experiments, we select the stiffness of MRE damper ranged $2.2 \times 10^{8} \mathrm{~N} / \mathrm{m}-4.1 \times 10^{8} \mathrm{~N} / \mathrm{m}$, which stiffness varies with its current relationship is shown in Table 1

Table 1 The stiffness of MRE damper

\begin{tabular}{cccc}
\hline electric current $/ \mathrm{A}$ & 0 & 0.5 & 1 \\
\hline stiffness $\times 10^{8} \mathrm{~N} / \mathrm{m}$ & 2.2 & 3.0 & 4.1 \\
\hline
\end{tabular}

Analysis control effect of MRE damper to the structure translational and torsional vibration by Simulink toolbox in the MATLAB, and analysis the damping effect while applying the optimal control force to the each layers of the structure. MRE vibration simulation module is shown in Figure 2, the optimal control simulation module is shown in Figure 3.

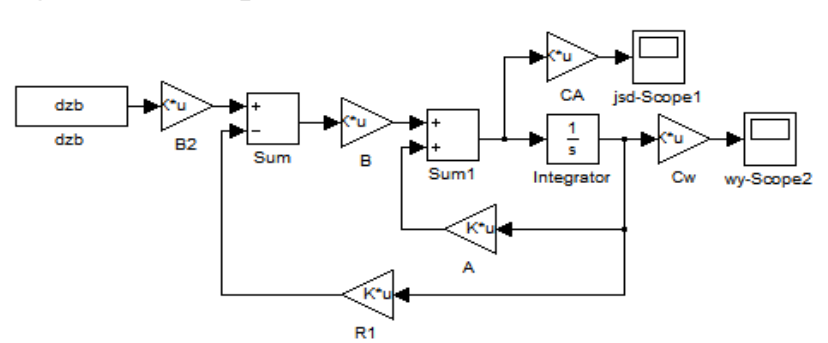

Fig. 2 MRE vibration simulation module

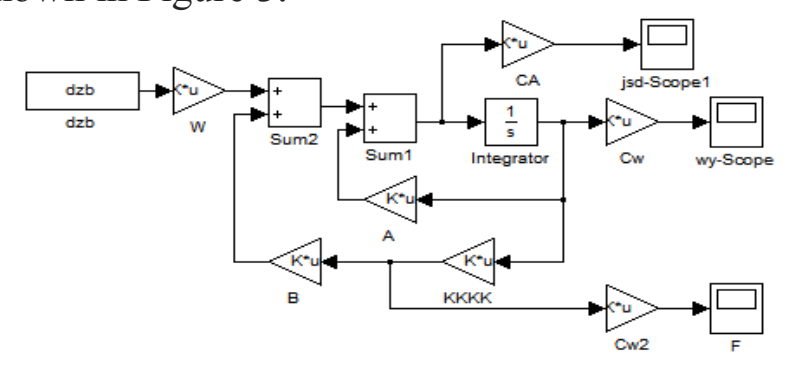

Fig. 3 the optimal control simulation module

Taking the top layer as an example, the time history analysis of the translational and torsional response of the top layer in the structure in the absence of control and with MRE damper control action are as shown in Figure 4 to 6 . The time history analysis of the translational and torsional response of the top layer in the structure in the absence of control and with optimal control are as shown in Figure 7 to 9 . The maximum of time history analysis of the translational and torsional response of the first layer and the top layer are as shown in Table 2 and Table 3. 


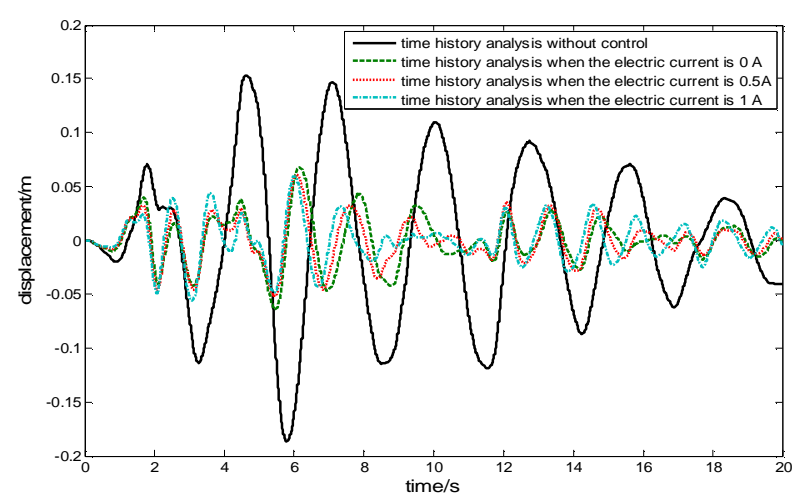

Fig. 4 time history analysis of the top layer to $\mathrm{X}$ direction

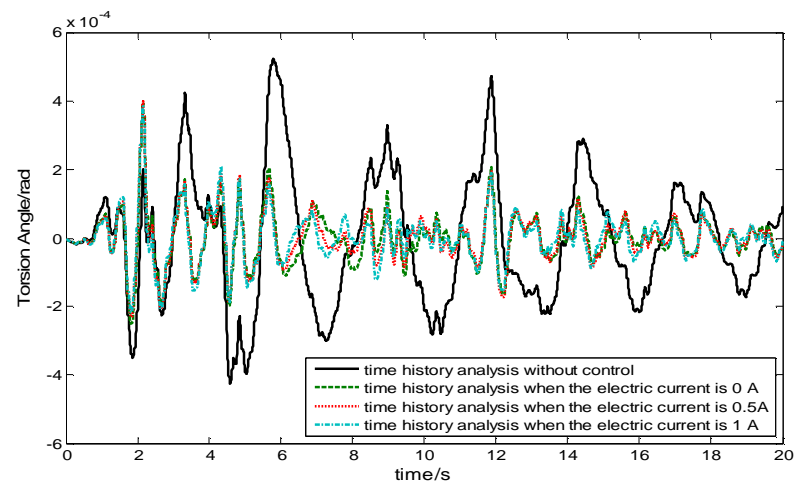

Fig. 6 time history analysis of torsional response of the top layer

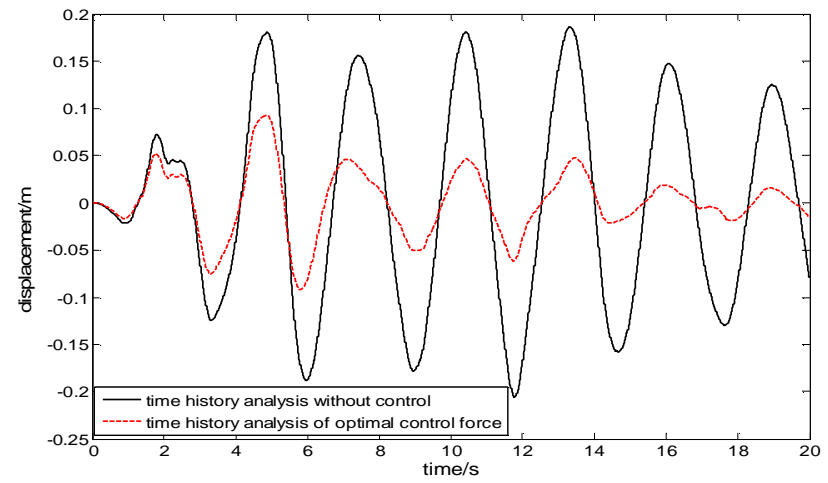

Fig. 8 time history analysis of the top layer to Y direction under optimal control

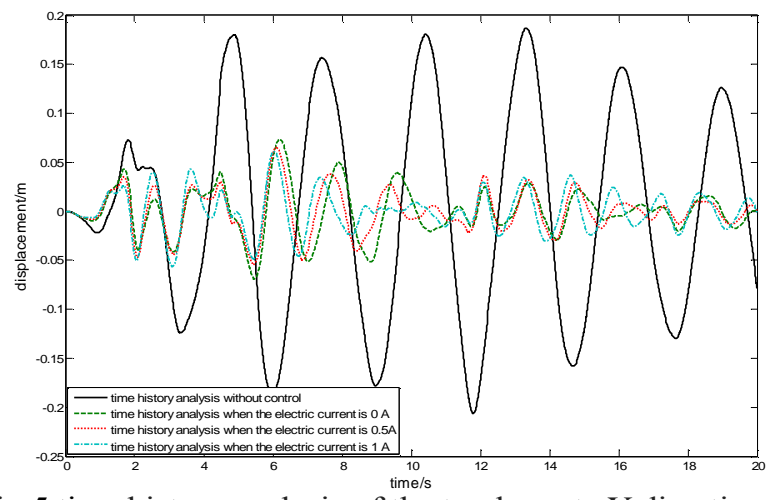

Fig.5 time history analysis of the top layer to Y direction

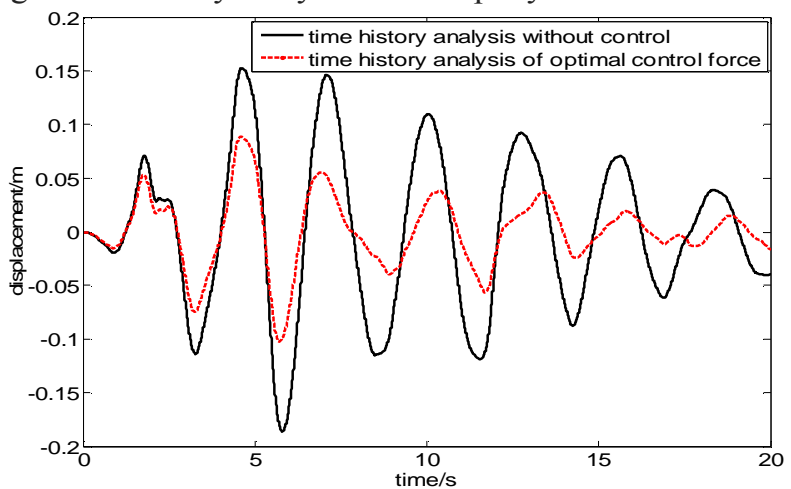

Fig. 7 time history analysis of the top layer to $\mathrm{X}$ direction under optimal control

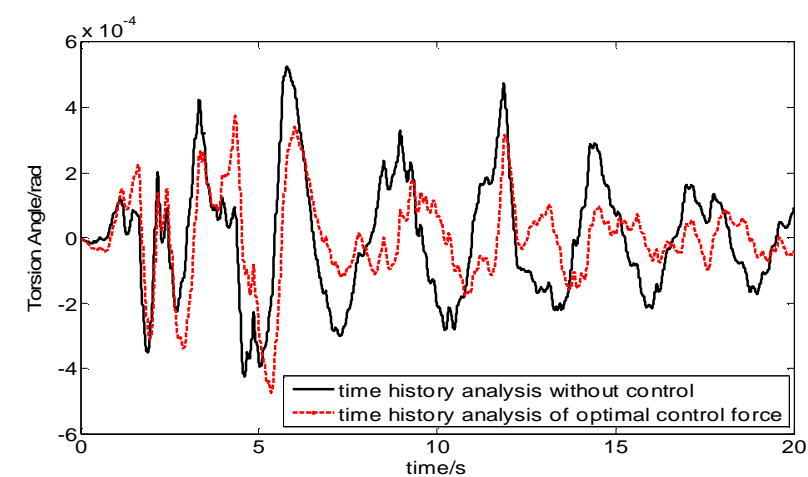

Fig.9 time history analysis of torsional response of the top layer under optimal control

Table 2 The maximum response contrast of the structure in the absence of control and with MRE damper control action

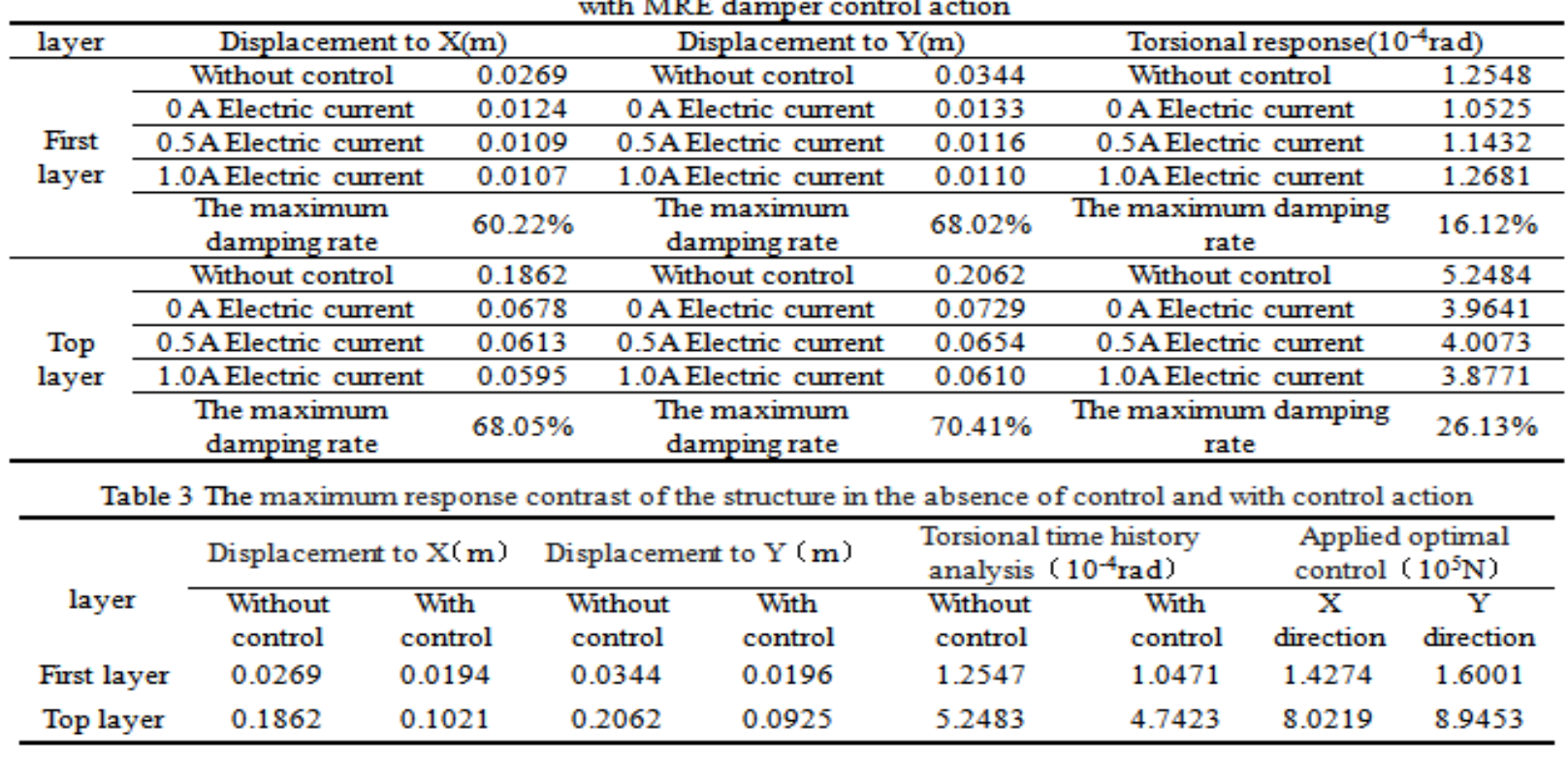




\section{Conclusion}

This article focuses on vibration control effect of MRE damper to the eccentric structure, and the conclusions are as follows: (1) It has better control effect of translational and torsional vibrations of the structure while installed MRE damper in the structure. From the results of numerical examples: the damping rate of the first layer to $\mathrm{X}$ direction can reach a maximum of $60.22 \%$, to $\mathrm{Y}$ direction can reach a maximum of $68.02 \%$, the torsional damping ratio can reach a maximum of $16.12 \%$; the damping rate of the top layer to $\mathrm{X}$ direction can reach a maximum of $68.05 \%$, to $\mathrm{Y}$ direction can reach a maximum of $70.41 \%$, the torsional damping ratio can reach a maximum of $26.13 \%$. The vibration control effect of the damper to $\mathrm{Y}$ direction is better than to $\mathrm{X}$ direction, on the top layer is better than the first floor. (2) As the input electric current increases, the magnetic field applied in the MRE is enhanced, the MRE dampers makes the translational vibration damping effect of the structure get better. The torsional vibration damping effect is not such a law. (3) Under the optimal control action, the translational and torsional vibrations of the structure have been better controlled.

\section{Acknowledgements}

The authors gratefully acknowledge the Natural Science Foundation of China (National Key Project No.51278393)

\section{References}

[1] Dang Yu,Huo Kaicheng.2010. Research on the Effect of Torsional Response of Base-isolated Buildings. Journal of Wuhan University of Technology,32(14):137-141.

[2] Xiao Mingkui, Zheng Meng, Chang Jisheng.2012.Analysis of combined seismic isolation response of irregular frame structures. Earthquake engineering and Engineering Vibration,32(4):174-181.

[3] Tang Yuguo, Deng Xuesong, Zhou Yun.2008.Research and Applications of Tortional Control of Eccentric Structure under Earthquake. Earthquake resistant and retrofit of Engineering,30(2):39-44.

[4] Ni, Y.Q. Ying, Z.G. Chen, Z.H. 2010.Magneto-rheological elastomer (MRE) based composite structures for micro-vibration control. Earthquake Engineering and Engineering Vibration, 9(3):345-356,

[5] Tu Jianwei, Qu Weilian, Zhou Chengming.2003. Test of MR Damper and RBF Network Model . Journal of Wuhan University of Technology, 25(1):43-45.

[6] Long, Ming, Hu, Guoliang, Wang, Shaolong.2013.Vibration response analysis of MRE cantilever sandwich beam under non-homogeneous magnetic fields. Applied Mechanics and Materials, 30(3): 49-52.

[7] Liu Jingbo,Du Xiuli.2005.Structure dynamics . Bei Jing: Press of machinery industry

[8] Fang Sheng, Gong Xinglong, Zhang Xianzhou.2004.Mechanical Analysis and Measurement of Magnetorheological Elastomers. Journal of University of Science \& Technology China,4(4):75-82.

[9] Fu Jie,Ju Benxiang, Yu Miao.2013.Influence of thickness of the sample on the mechanical properties of magnetorheological elastomers. Functional material,44(9):1277-1280.

[10]Xu Jiayun, Jian Yuanping, Chang Yinchang.2007.Vibration control of bridge with magneto-rheological dampers. Journal of natural disasters, 16 (4) :82-85.

[11]Zhou Chencheng.2014.Study on the magnetic rheological elastomer and its application in the semi-active control of structures, Nan Jing: Nanjing University of Science and Technology. 